\title{
Determinación de cadmio y plomo en agua, sedimento y organismos bioindicadores en el Estero Salado, Ecuador
}

\section{(Determination of cadmium and lead in water, sediment and bioindicator organisms in Estero Salado, Ecuador)}

\author{
Beatriz Pernía ${ }^{1}$, Mariuxi Mero², Xavier Cornejo ${ }^{3}$, Nelson Ramírez ${ }^{1}$, Lissette Ramírez ${ }^{1}$, \\ Kenya Bravo ${ }^{1}$, David López ${ }^{1}$, Jorge Muñoz ${ }^{1}$, Josué Zambrano ${ }^{1}$
}

\begin{abstract}
Resumen:
Existen evidencias de contaminación por metales pesados en el Estero Salado, localizado en la ciudad de Guayaquil, provincia del Guayas, en la costa de Ecuador. Con la finalidad de identificar organismos bioindicadores de contaminación por metales pesados, se estudió la concentración de $\mathrm{Cd}$ y $\mathrm{Pb}$ en agua, sedimento, hojas de mangles como Rhizophora racemosa, Avicennia germinans, Conocarpus erectus y Laguncularia racemosa, y gasterópodos como Littorina varia y Cerithidea mazatlanica, que se encontraban a nivel del Puente Portete en el Estero Salado. Las muestras fueron analizadas por espectrofotometría de absorción atómica de flama. Los valores de Cd y $\mathrm{Pb}$ en agua y sedimento superaron a los límites permisibles establecidos en las normas ecuatoriana y canadiense. Los gasterópodos $L$. varia y $C$. mazatlanica demostraron ser los mejores bioindicadores de contaminación al acumular en sus tejidos blandos $2,45 \pm 0,49 \mathrm{mg} \mathrm{kg}^{-1}$ de $\mathrm{Pb}$ y $1,49 \pm 0,67 \mathrm{mg} \mathrm{kg}^{-1} \mathrm{de} \mathrm{Cd}$, respectivamente. Los mangles no acumularon en sus hojas $\mathrm{Cd}$ y $\mathrm{Pb}$ por la baja biodisponibilidad en el sedimento.
\end{abstract}

Palabras clave: centinelas; metales pesados; Puente Portete; Littorina varia; Cerithidea mazatlanica

\begin{abstract}
:
Evidence of pollution by heavy metals have been detected in the city of Guayaquil, province of Guayas, coastal Ecuador. The water and sediment in the mangrove environs of Puente Portete in Estero Salado were tested for concentrations of cadmium $(\mathrm{Cd})$ and lead $(\mathrm{Pb})$, as well as the following organisms: gastropods, including Littorina varia and Cerithidea mazatlanica, and the leaves of Rhizophora racemosa, Laguncularia racemosa, Avicennia germinans, and Conocarpus erectus. The samples were analyzed using flame atomic absorption spectrophotometry. The values of $\mathrm{Cd}$ and $\mathrm{Pb}$ in water and sediment exceeded the permissible limits established by Ecuadorian and Canadian standards. The gastropod species $L$. varia and C. mazatlanica were the best indicator organisms for detecting pollution by accumulating $2.45 \pm 0.49 \mathrm{ppm} \mathrm{Pb}$ and $1.49 \pm 0.67 \mathrm{ppm} \mathrm{Cd}$, respectively. Because of the low bioavailability of heavy metals in sediments, mangrove plants did not accumulate $\mathrm{Cd}$ and $\mathrm{Pb}$ in leaves.
\end{abstract}

Keywords: sentinels; heavy metals; Portete bridge; Littorina varia; Cerithidea mazatlanica

\footnotetext{
1 Facultad de Ciencias Naturales, Universidad de Guayaquil, Guayaquil, Ecuador (beatriz.pernias@ug.edu.ec).

${ }^{2}$ Instituto de Investigaciones de Recursos Naturales, Universidad de Guayaquil, Guayaquil, Ecuador (Mariuxi.merov@ug.edu.ec).

${ }^{3}$ Herbario GUAY, Facultad de Ciencias Naturales, Universidad de Guayaquil, Guayaquil, Ecuador (Xavier.cornejos@ug.edu.ec).
} 


\section{Introducción}

El Estero Salado se encuentra localizado en el estuario interno del Golfo de Guayaquil, que abarca el $81 \%$ de los manglares del Ecuador; alberga una variedad de peces, crustáceos y moluscos de valor ecológico y comercial (Monserrate et al., 2011). El Estero Salado recorre parte de Guayaquil, la ciudad más poblada del Ecuador, la cual en los últimos años ha sufrido un grave deterioro de la calidad de sus aguas como consecuencia de las actividades antropogénicas, descargas de aguas residuales domésticas, agrícolas e industriales, las cuales son vertidas sin ningún tipo de tratamiento. Como consecuencia, este estuario se ha convertido en un reservorio de diversos contaminantes, entre los que destacan los metales pesados (Monserrate et al., 2011; Fernández-Cadena et al., 2014).

De todos los contaminantes, los metales pesados han recibido una atención especial debido a que no son biodegradables, son tóxicos en bajas concentraciones y tienden a acumularse en las zonas costeras y estuarinas (Lacerda et al., 1988). Además de ello, son acumulados en los organismos acuáticos donde se genera un proceso de bioacumulación y bioamagnificación (Pernía et al., 2008).

De los metales pesados, el $\mathrm{Cd}$ y el Pb son de los más tóxicos. La exposición a Cd se ha asociado a diversas enfermedades renales (Suwazono et al., 2006), hipertensión (Gallagher y Meliker, 2010), anemia, osteoporosis (Järup y Alfvén, 2004), osteomalacia (WHO, 2010), diabetes (Schwartz et al., 2003), anosmia, rinitis crónica, eosinofilia (Henson y Chedrese, 2004) y algunos tipos de cáncer, tales como: leucemia (Henson y Chedrese, 2004), cáncer de mama (McElroy et al., 2006), páncreas, pulmones (Henson y Chedrese, 2004) y próstata (Julin et al., 2012). En peces, aves y mamíferos genera reducción de crecimiento, anemia, hipertensión disrupciones endócrinas y en anfibios decrecimiento de la metamorfosis (Richardson et al., 1974; Revis et al., 1981; Vásquez et al., 2005; Burger, 2008).

Además, el $\mathrm{Pb}$ genera alteraciones en el estatus iónico celular y estrés oxidativo, lo que trae como consecuencia disrupciones endócrinas, inhibición de la actividad enzimática, muerte celular, genotoxicidad y problemas inflamatorios (Lassiter et al., 2015). También se ha atribuido al $\mathrm{Pb}$ la inhibición de la síntesis de hemoglobina, generando anemia (Counter et al., 2012), incremento de la presión sanguínea, daño renal (Batuman et al., 1981; Poma, 2008), perturbaciones en el sistema nervioso central y cáncer de estómago y pulmones (Mushak, 2011). Además, los niños son muy sensibles al Pb, el cual genera retraso en el desarrollo mental e intelectual (Poma, 2008). En los animales el Pb genera retardos en la maduración, afecta la cantidad y calidad de la esperma y produce cambios conductuales en mamíferos, invertebrados y en las aves puede ser mortal a bajas concentraciones (Lassiter et al., 2015).

Algunos estudios han mostrado el alto contenido de $\mathrm{Cd}$ y $\mathrm{Pb}$ en agua y sedimento dentro del Estero Salado, en el área de Puente Portete (Alcívar y Mosquera, 2011; Jiménez, 2012; Rodríguez, 2013), los cuales permiten tener valores referenciales del comportamiento de estos en la zona de estudio a través de los años.

En la presente investigación se plantea el uso de organismos como complemento a los ensayos físicos y químicos, ya que estos permiten estudiar la biodisponibilidad de los contaminantes. Estos organismos se han denominado bioindicadores o centinelas y se definen como organismos que nos aportan información sobre la calidad del ambiente, mediante la detección de algún contaminante (Castrillo et al., 2012).

Es por ello que los objetivos planteados en esta investigación fueron evaluar las concentraciones de cadmio y plomo totales en agua y sedimentos en el Estero Salado, sector el Portete; estudiar la biodisponibilidad de los metales pesados en el sedimento y evaluar el efecto de los manglares sobre la biodisponibilidad e identificar organismos bioindicadores de contaminación por estos metales pesados en la zona de estudio. 


\section{Metodología}

\section{Área de Estudio}

El Estero Salado es un sistema estuarino situado al occidente del río Guayas y al oeste del Golfo de Guayaquil, Ecuador, el cual se ramifica hasta la ciudad de Guayaquil. El presente trabajo se realizó a nivel del puente Portete, ubicado entre las calles Portete y Barcelona, al suroeste de la ciudad de Guayaquil.

El estudio se llevó a cabo desde agosto de 2014 hasta agosto de 2015. Se seleccionaron un total de ocho estaciones de muestreo: cuatro en zonas de manglar (M1, M2, M3 y M4) y cuatro cercanas al puente donde no había presencia de vegetación (P1, P2, P3 y P4) a fin de determinar el efecto del manglar sobre la biodisponibilidad de los metales en el sedimento. Las coordenadas de cada punto de muestreo fueron determinadas utilizando un GPS modelo Triton (Magellan®). (Tabla 1)

En cada estación se captaron muestras de agua superficial, sedimentos y organismos, en período de marea baja, en época de sequía, ya que en la época lluviosa los niveles de concentración de los metales pesados disminuyen como consecuencia del efecto de dilución que genera la entrada de agua dulce al estuario (Campos y Gallo, 1997; Peñafiel et al., 2017).

Tabla 1. Coordenadas geográficas de los puntos de muestreo en el Estero Salado.

\begin{tabular}{|l|c|c|}
\hline Puntos & W & S \\
\hline M1 & $79^{\circ} 17^{\prime} 61^{\prime \prime}$ & $2^{\circ} 19^{\prime} 54^{\prime \prime}$ \\
\hline M2 & $79^{\circ} 93^{\prime} 75^{\prime \prime}$ & $2^{\circ} 19^{\prime} 54^{\prime \prime}$ \\
\hline M3 & $79^{\circ} 93^{\prime} 76^{\prime \prime}$ & $2^{\circ} 19^{\circ} 54$ \\
\hline M4 & $79^{\circ} 93^{\prime} 77^{\prime \prime}$ & $2^{\circ} 19^{\circ} 54$ \\
\hline P1 & $79^{\circ} 93^{\prime} 71^{\prime \prime}$ & $2^{\circ} 19^{\circ} 54$ \\
\hline P2 & $79^{\circ} 93^{\prime} 68^{\prime \prime}$ & $2^{\circ} 19^{\circ} 54$ \\
\hline P3 & $79^{\circ} 93^{\prime} 68^{\prime \prime}$ & $2^{\circ} 19^{\circ} 53$ \\
\hline P4 & $79^{\circ} 93^{\prime} 69^{\prime \prime}$ & $2^{\circ} 19^{\circ} 54$ \\
\hline
\end{tabular}

\section{Parámetros fisicoquímicos}

Los parámetros $\mathrm{pH}$, temperatura y salinidad, fueron determinados in situ con equipos calibrados: YSI pH10 (EcoSense®) y ATC/mil-E (Atago®).

\section{Determinación de metales pesados en agua}

Las muestras de agua se colectaron con un muestreador tipo Cazo, por triplicado y colocadas en botellas de polietileno de alta densidad de $1000 \mathrm{~mL}$ previamente tratadas durante $24 \mathrm{~h}$ con $\mathrm{HNO}_{3}$ al $3 \%$ y lavadas con agua ultra pura, según la norma COVENIN 2709:2002. Las muestras fueron acidificadas $(\mathrm{pH}<2)$ utilizando $1 \mathrm{~mL}$ de $\mathrm{HNO}_{3}$ concentrado (Merck) y trasladadas hasta el laboratorio. Después las muestras fueron filtradas por triplicado utilizando papel Whatman №40 y el Cd y Pb total fue detectado en el agua.

\section{Determinación de metales pesados biodisponibles y totales en sedimentos}

Los sedimentos se tomaron de la capa superficial, $5-10 \mathrm{~cm}$ de profundidad, con una espátula de plástico y se conservaron en fundas de cierre hermético hasta llegar al laboratorio. Una vez en el laboratorio se procesaron según la metodología propuesta por Mero et al., (2012). Los sedimentos fueron extendidos sobre una superficie plástica y secados a temperatura ambiente durante dos semanas. Posteriormente, fueron disgregados y tamizados a través de un tamiz de $71 \mu \mathrm{m}$. Las muestras tamizadas se secaron en una estufa a $80^{\circ} \mathrm{C}$ por $1 \mathrm{~h}$ para eliminar la humedad y se pesó $1 \mathrm{~g}$ para su posterior digestión por triplicado. 
Para la detección de la fracción total de metales, se utilizó un procedimiento validado a partir de la Norma EPA3050B. $1 \mathrm{~g}$ de muestra fue digerido con $5 \mathrm{~mL}$ de $\mathrm{HNO}_{3}$ en tubos de ensayos herméticamente sellados, en baño maría a $100^{\circ} \mathrm{C}$ por $1 \mathrm{~h}$. Posteriormente, se filtró con papel Whatman $\mathrm{N}^{\circ} 40$ y se enrasó a $50 \mathrm{~mL}$ con agua ultrapura.

Para la detección de la fracción de metales biodisponibles, se utilizó la metodología utilizada por Parra y Espinosa (2008). A $1 \mathrm{~g}$ de sedimento seco se le añadieron $15 \mathrm{~mL}$ de $\mathrm{HCl} 0,1 \mathrm{~N}$ y se dejó en reposo durante $24 \mathrm{~h}$, se filtró con papel Whatman $\mathrm{N}^{\circ} 40$ y se enrasó a $50 \mathrm{~mL}$ con el mismo ácido. Como control de calidad se utilizó material de referencia certificado (CRM-016 Trace metals/Fresh water sediment) con un porcentaje de recuperación de $99 \pm 1 \%$.

\section{Identificación de plantas y gasterópodos comunes}

Se realizó una inspección visual de la zona de estudio a fin de definir cuáles eran las especies de mangle e invertebrados presentes en mayor abundancia. Las muestras fueron identificadas por taxonomía clásica utilizando para ello claves taxonómicas (Cornejo, 2014).

\section{Determinación de $\mathrm{Cd}$ y $\mathrm{Pb}$ en mangles}

Para la toma de muestras más abundantes de la flora se utilizó una transecta lineal. Se tomaron 20 hojas por individuo, utilizando guantes para no contaminar el material y se colocaron en bolsas plásticas transparentes con cierre hermético. Una vez transportadas al laboratorio se lavaron con agua destilada, se ubicaron en cápsulas de porcelana, se secaron en una estufa a $80^{\circ} \mathrm{C}$ por $48 \mathrm{~h}$, se trituraron en un mortero y se pesó $1 \mathrm{~g}$ para su posterior digestión por triplicado.

A $1 \mathrm{~g}$ de hoja se añadieron $5 \mathrm{~mL}$ de $\mathrm{HNO}_{3}$, se calentó a $60^{\circ} \mathrm{C}$, hasta la eliminación de humos marrones o rojos, se añadió $1 \mathrm{~mL}$ de $\mathrm{H}_{2} \mathrm{O}_{2}$ y una vez digerida la muestra se filtró a través de papel Whatman $\mathrm{N}^{\circ} 40$ y se enrasó a $50 \mathrm{~mL}$ con agua ultrapura.

\section{Determinación de $\mathrm{Cd}$ y $\mathrm{Pb}$ en invertebrados}

Los organismos hallados en mayor abundancia fueron dos especies de gasterópodos, por lo que se utilizó la metodología propuesta por Alcívar y Mosquera (2011). Las muestras de gasterópodos $(n=30)$ por punto de muestreo, se guardaron en fundas plásticas en una hielera a $4^{\circ} \mathrm{C}$ hasta llegar al laboratorio, se lavaron con agua destilada para eliminar restos de sedimento. Se retiró el cuerpo de los gasterópodos partiendo la concha con un mortero. Posteriormente, los cuerpos se colocaron en cápsulas de porcelana y se secaron en estufa (MEMMERT) a $80^{\circ} \mathrm{C}$ durante $48 \mathrm{~h}$, hasta obtener peso constante. Luego se trituraron en un mortero, se llevaron a estufa por $1 \mathrm{~h}$ y se pesó $1 \mathrm{~g}$ en una balanza analítica modelo BL210S (Sartorius), se añadieron $5 \mathrm{~mL}$ de $\mathrm{HNO}_{3}$ (Merck) y se dejó digerir durante $24 \mathrm{~h}$ a $100^{\circ} \mathrm{C}$, hasta eliminar humos marrones o rojos y una vez digerida la muestra se filtró a través de papel Whatman $\mathrm{N}^{\circ} 40$ y se enrasó a $25 \mathrm{~mL}$ con agua ultrapura.

\section{Cuantificación de $\mathrm{Cd}$ y Pb}

La cuantificación de $\mathrm{Cd}$ y $\mathrm{Pb}$ de las muestras de agua, sedimento y organismos se realizó por la técnica de espectrofotometría de absorción atómica de llama, utilizando un espectrofotómetro modelo AAnalyst100 (Perkin Elmer). Para el control de calidad el equipo fue calibrado con estándares certificados (Accustandar). Se utilizó material de referencia para sedimentos (CRM-016 Trace metals/Fresh water sediment) como control de calidad y las lecturas de las muestras se realizaron por triplicado para verificar su repetibilidad. 


\section{Análisis estadísticos}

El muestreo se realizó de manera aleatoria. Se determinó la normalidad de los datos utilizando una prueba de Anderson-Darling y homocedasticidad mediante un test de Levene. Para comparar las medias de los parámetros y concentraciones de $\mathrm{Cd}$ en los distintos sitios de muestreo se aplicó una ANOVA de una vía, tomando $\mathrm{P}<0.05$ como valor significativo y un test a posteriori de Tukey. En los casos donde no hubo normalidad se aplicó una prueba no paramétrica de Kruskall-Wallis.

Además, se realizó un análisis de componentes principales y un análisis de correlación de Pearson para evidenciar la influencia de los parámetros fisicoquímicos sobre la concentración de $\mathrm{Cd}$ y $\mathrm{Pb}$ en agua, sedimento y organismos. Las pruebas estadísticas se realizaron utilizando el programa MINITAB versión 17.0.

\section{Resultados}

La concentración promedio de Cd en agua en la zona de manglar fue de 0,053 $\pm 0,001$ $\mathrm{mg} \mathrm{L}^{-1}$ y no hubo diferencias significativas entre los cuatro puntos de muestreo (Figura 1A). Sin embargo, la concentración fue significativamente mayor $(H=20,73, P=0,004)$ en la zona del puente con un promedio de 0,072 $\pm 0,006 \mathrm{mg} \mathrm{L}^{-1}$. La concentración de $\mathrm{Pb}$ no mostró un patrón entre ambas zonas, sin embargo, se observaron las mayores concentraciones en la zona del puente en los puntos $\mathrm{P} 1=0,264 \pm 0,002 \mathrm{mg} \mathrm{L}^{-1}$ y P2 $=0,228 \pm 0,024 \mathrm{mg} \mathrm{L}^{-1}$ $(F=12,67, P=0,000$; Fig. 1B).

A.
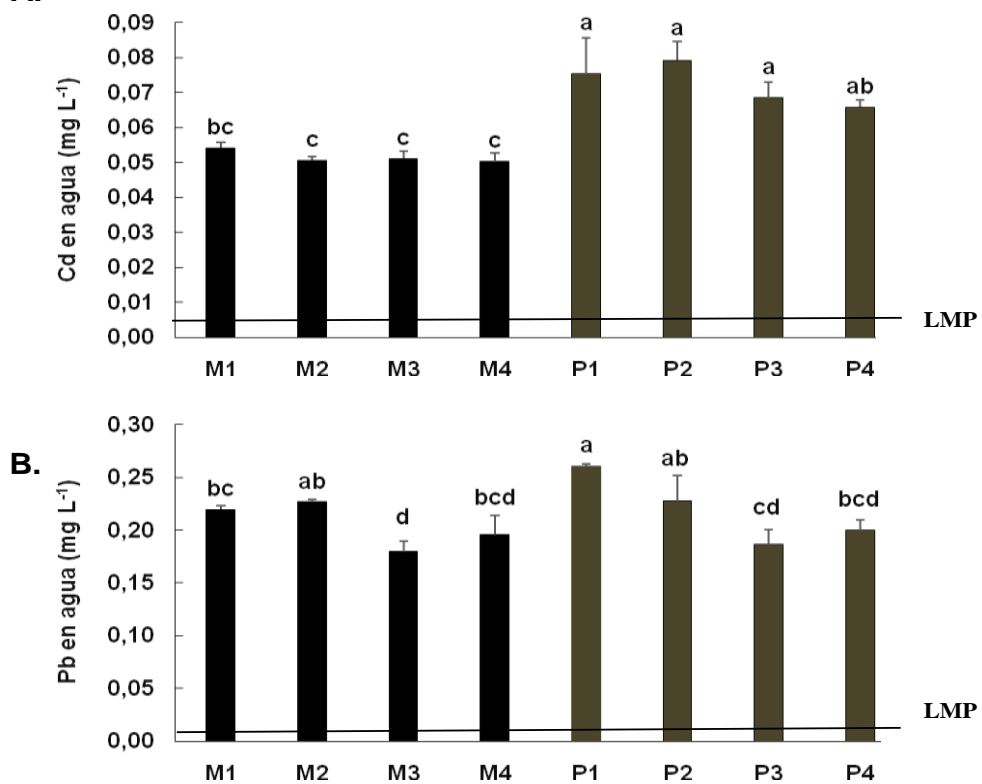

Figura 1. Concentración de A. Cd y B. Pb en agua en el Estero Salado a nivel de Puente Portete. $\mathbf{M}$ : representan las muestras tomadas en la zona de manglar y $\mathbf{P}$ : las muestras tomadas en la zona del puente, LMP: límite máximo permitido según TULAS y OMS. Los resultados se muestran como barras que representan la media $\pm D S,(n=3)$. Letras iguales señalan que no hay diferencias estadísticamente significativas según Kruskal Wallis o ANOVA de 1-vía $(P>0.05)$ y test de Tukey.

En los sedimentos los valores fueron mayores a los presentes en el agua en las dos zonas (Figura 2A). El Cd se encontró a una concentración promedio para la zona $\mathrm{M}=$ $0,106 \pm 0,132 \mathrm{mg} \mathrm{kg}^{-1}$. Además, en la zona $P$ la concentración de Cd fue $2341,9 \pm 0,47 \mathrm{mg}$ $\mathrm{kg}^{-1}$ que supera 2.8 veces al límite máximo permitido para sedimentos según la Norma Internacional de Canadá "Canadian Sediment Quality Guidelines", (1999), la cual establece un máximo de $0,676 \mathrm{mg} \mathrm{kg}^{-1}$ de $\mathrm{Cd}$ en sedimentos. 
A.
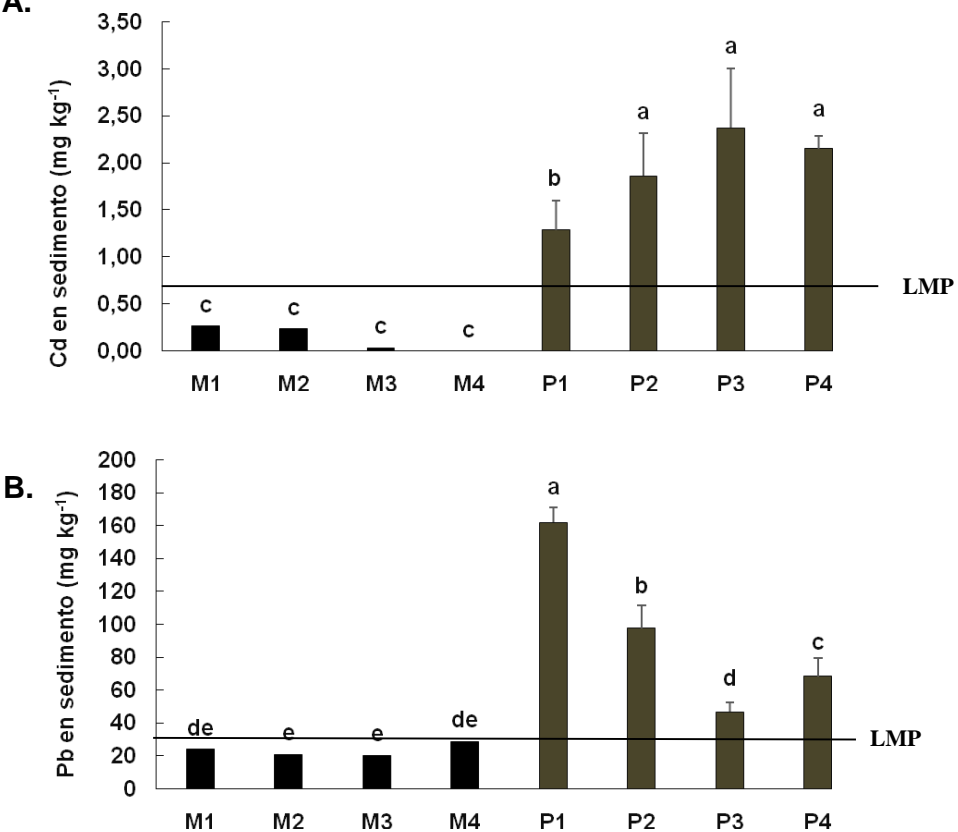

Figura 2. Concentración de A. Cd y B. Pb en sedimentos del Estero Salado a nivel de Puente Portete. M: representan las muestras tomadas en la zona de manglar y P: las muestras tomadas en la zona del puente. LMP: límite máximo permitido según TULAS y OMS. Los resultados se muestran como barras que representan la media $\pm D S,(n=3)$. Letras iguales señalan que no hay diferencias estadísticamente significativas según Kruskal Wallis o ANOVA de 1-vía $(P>0.05)$ y test de Tukey.

El $\mathrm{Pb}$ en los sedimentos presentó el mismo patrón que el $\mathrm{Cd}$, encontrándose valores permisibles en la zona $\mathrm{M}$ y valores altos en la zona $\mathrm{P}$ (Figura $2 B$ ). En la zona $\mathrm{M}$, no se observaron diferencias significativas entre los cuatro puntos muestreados, mostrando una media de $23,57 \pm 3,87 \mathrm{mg} \mathrm{kg}^{-1} \mathrm{~Pb}$. Sin embargo, en la zona $\mathrm{P}$ se determinaron diferencias significativas $(F=125,55, P=0,000)$, entre los cuatro puntos en el siguiente orden: $(P 1=$ $\left.162,21 \pm 9,277 \mathrm{mg} \mathrm{kg}^{-1} \mathrm{~Pb}\right)>\left(\mathrm{P} 2=97,81 \pm 14,10 \mathrm{mg} \mathrm{kg}^{-1} \mathrm{~Pb}\right)>\left(\mathrm{P} 4=68,65 \pm 11,23 \mathrm{mg} \mathrm{kg}^{-1}\right.$ $\mathrm{Pb})>\left(\mathrm{P3}=47,03 \pm 5,85 \mathrm{mg} \mathrm{kg}^{-1} \mathrm{~Pb}\right)$.

Además, en cuanto a la biodisponibilidad de los metales en el sedimento se evidenció que en la zona $\mathrm{M}$ el $\mathrm{Cd}$ no se encontraba biodisponible, mientras que en la zona $\mathrm{P}$ por el contrario, el Cd se encontró biodisponible en un 70,82-89,47\% (Figura 3). En el caso del $\mathrm{Pb}$ si se encontró biodisponible tanto en la zona $\mathrm{M}$ como en $\mathrm{P}$ con valores promedio de $77,41 \%$ y $71,64 \%$, respectivamente. Para verificar la hipótesis inicial donde se afirma que los manglares afectan la biodisponibilidad de los metales se realizó una prueba de KruskallWallis y se demostró que la biodisponibilidad de $\mathrm{Cd}$ se ve afectada por la presencia de los manglares $(H=5,33, P=0,021)$, mientras que la biodisponibilidad del $\mathrm{Pb}$ no $(H=0,08$, $\mathrm{P}=0,773)$. 


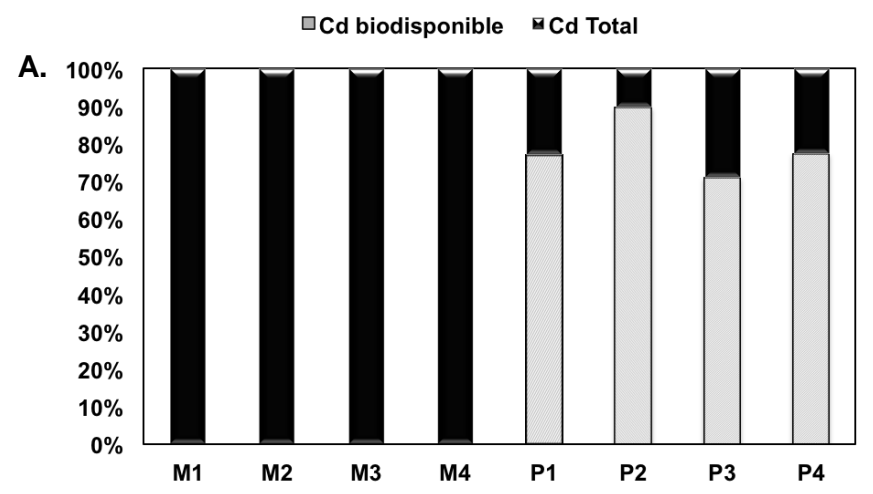

B.

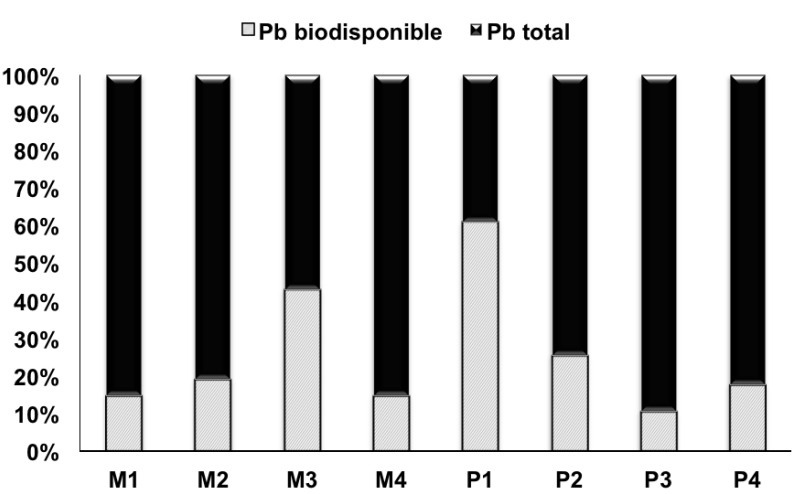

Figura 3. Comparación de los porcentajes de biodisponibilidad de los distintos metales en los sedimentos. A. Cd y B. Pb. M: representan las muestras tomadas en la zona de manglar y $\mathbf{P}$ : las muestras tomadas en la zona del puente.

Parámetros fisicoquímicos: los parámetros fisicoquímicos estudiados mostraron que las zonas de manglar $(M)$ y Puente $(P)$ solo presentaron diferencias en la temperatura, los otros parámetros fisicoquímicos no presentaron variaciones significativas (Tabla 2).

Tabla 2. Variables fisicoquímicas: temperatura, $\mathrm{pH}$ y salinidad de los puntos de muestreo.

\begin{tabular}{|l|l|l|l|}
\hline Puntos & $\mathbf{T}\left(\mathbf{C}^{\circ}\right)$ & $\mathbf{p H}$ & $\begin{array}{l}\text { Salinidad } \\
(\%)\end{array}$ \\
\hline M1 & 27,9 & 7,45 & 25 \\
\hline M2 & 27,5 & 7,55 & 23 \\
\hline M3 & 26,6 & 7,60 & 23 \\
\hline M4 & 26,7 & 7,50 & 23 \\
\hline P1 & 28,0 & 7,40 & 24 \\
\hline P2 & 29,0 & 7,47 & 20 \\
\hline P3 & 28,2 & 7,73 & 25 \\
\hline P4 & 28,9 & 7,75 & 25 \\
\hline Promedio M & $27,17 \pm 0,63^{\mathrm{A}}$ & $7,53 \pm 0,06^{\mathrm{A}}$ & $23,50 \pm 1,00^{\mathrm{A}}$ \\
\hline Promedio P & $28,53 \pm 0,50^{\mathrm{B}}$ & $7,65 \pm 0,16^{\mathrm{A}}$ & $23,50 \pm 2,38^{\mathrm{A}}$ \\
\hline
\end{tabular}

La temperatura promedio fue de $27,17 \pm 0,63^{\circ} \mathrm{C}\left(26,6^{\circ} \mathrm{C}\right.$ mín, $27,9^{\circ} \mathrm{C}$ máx), en la zona de manglar y de $28,53 \pm 0,50^{\circ} \mathrm{C}\left(28^{\circ} \mathrm{C}\right.$ mín, $29^{\circ} \mathrm{C}$ máx $)$ en la zona del Puente, con diferencias significativas entre ambas zonas ( $\mathrm{F}=11,30, \mathrm{P}=0,015$; Tabla 2).

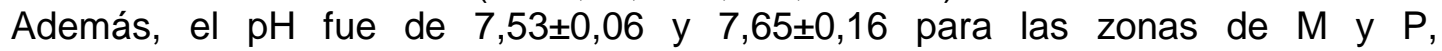
respectivamente, sin embargo, las diferencias no fueron estadísticamente significativas $(\mathrm{H}=0,27, \mathrm{P}=0,603)$. 
La salinidad promedio fue igual para las dos localidades $23,50 \%$ (Tabla 2), con una variación de $23-25 \%$ en la zona $\mathrm{M}$ y de $20-25 \%$ en la zona $\mathrm{P}$.

Organismos Bioindicadores: dentro de la zona de estudio $P$ no se observaron organismos y por el contrario, en la zona $\mathrm{M}$, se detectó la presencia de los mangles Rhizophora racemosa, Avicennia germinans, Conocarpus erectus y Laguncularia racemosa y de los gasterópodos Littorina varia y Cerithidea mazatlanica (Figura 4).

En cuanto al contenido de metales pesados en hojas, los manglares no acumularon $\mathrm{Cd}$ ni $\mathrm{Pb}$. Por el contrario, los gasterópodos $L$. varia y C. mazatlanica demostraron ser los mejores indicadores de contaminación, ya que acumularon $\mathrm{Cd}$ y $\mathrm{Pb}$ en altas concentraciones (Figura 4). L. varia mostró concentraciones de $2,45 \pm 0,49 \mathrm{mg} \mathrm{kg}^{-1}$ de Cd y $18,28 \pm 4,36 \mathrm{mg} \mathrm{kg}^{-1}$ de Pb y C. mazatlanica $1,49 \pm 0,67 \mathrm{mg} \mathrm{kg}^{-1} \mathrm{Cd}$ y $10,84 \pm 5,68 \mathrm{mg} \mathrm{kg}^{-1} \mathrm{de}$ $\mathrm{Pb}$. Comparativamente, $L$. varia fue el organismo que presentó los metales en mayor proporción, sin embargo C. mazatlanica fue la más abundante en la zona por lo que se recomienda utilizarla para fines de biomonitoreo.
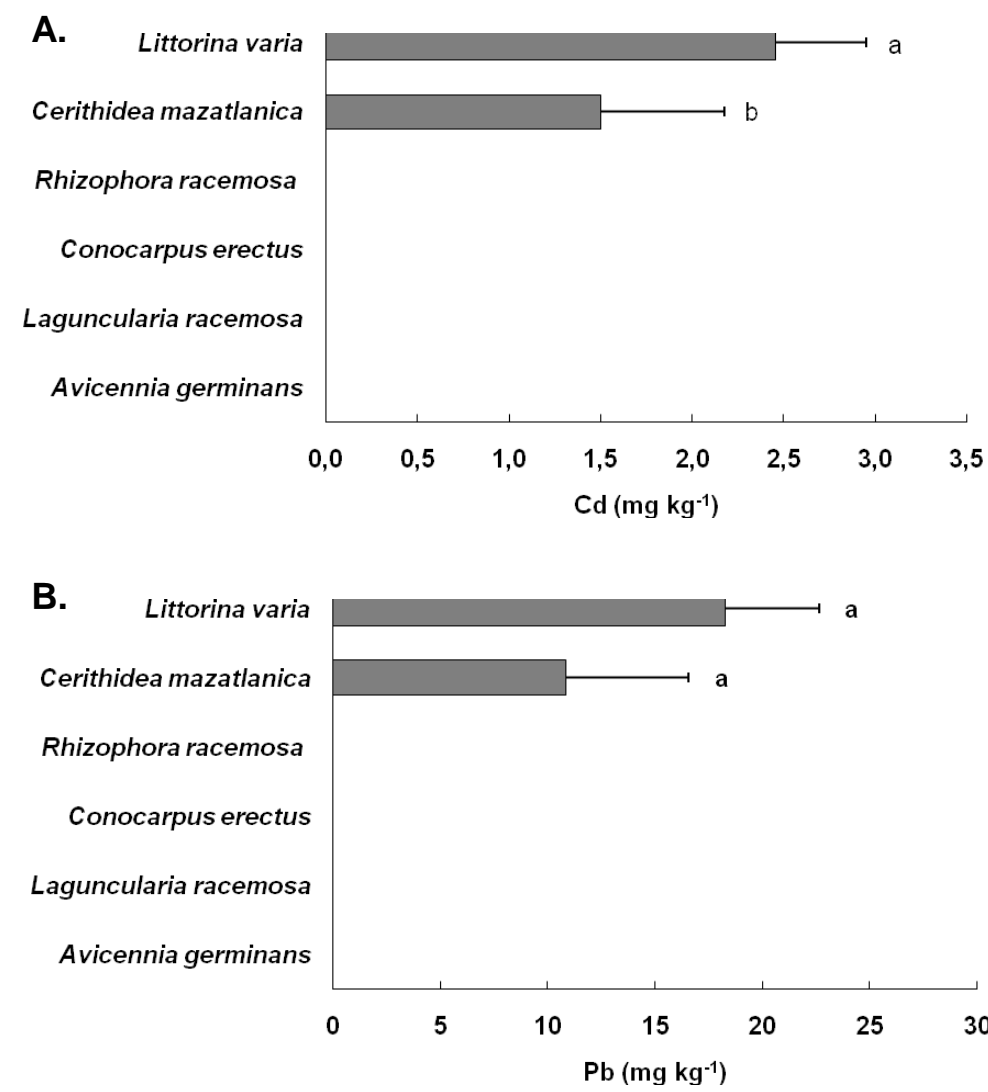

Figura 4. Concentración de $\mathrm{Cd}$ y $\mathrm{Pb}$ en los distintos bioindicadores: los gasterópodos Littorina varia y Cerithidea mazatlanica, y los mangles Rhizophora racemosa, Conocarpus erectus, Laguncularia racemosa y Avicennia germinans. Los resultados se muestran como medias \pm desviación estándar de $n=10$. Letras iguales señalan que no hay diferencias estadísticamente significativas entre las medias, según ANOVA de 1-vía $(P>0.05)$ y test de Tukey.

Además, se determinó correlación entre la concentración de Cd en los gasterópodos y el $\mathrm{Cd}$ biodisponible en el sedimento $(\mathrm{r}=1 ; P=0.000)$, al igual que entre el $\mathrm{Pb}$ en los organismos y el biodisponible $(r=0.688 ; P=0.013)$, por lo que estos organismos se pueden considerar indicadores de la biodisponibilidad de cadmio y plomo en el sedimento. 
Finalmente se evidenció el efecto de los parámetros fisicoquímicos sobre la biodisponibilidad de los metales y su influencia en la absorción por parte de los organismos. En el análisis de componentes principales se evidenció que cuatro componentes explicaron el $92 \%$ de la correlación entre las variables. Se apreció en el CP1 la correlación que existe entre la temperatura y la salinidad con la concentración de $\mathrm{Cd}$ y $\mathrm{Pb}$ en agua; de igual manera se apreció en el CP4 una correlación entre el Pb biodisponible en el sedimento con el presente en los gasterópodos (Figura 5). Para confirmar estos resultados se realizó un análisis de correlación de Pearson donde se evidenció correlación entre la concentración de $\mathrm{Pb}$ en agua y la temperatura $(\mathrm{r}=0,819, P=0,001)$ y el $\mathrm{Pb}$ en sedimento con el $\mathrm{pH}(\mathrm{r}=-$ $0.580, P=0,048)$.

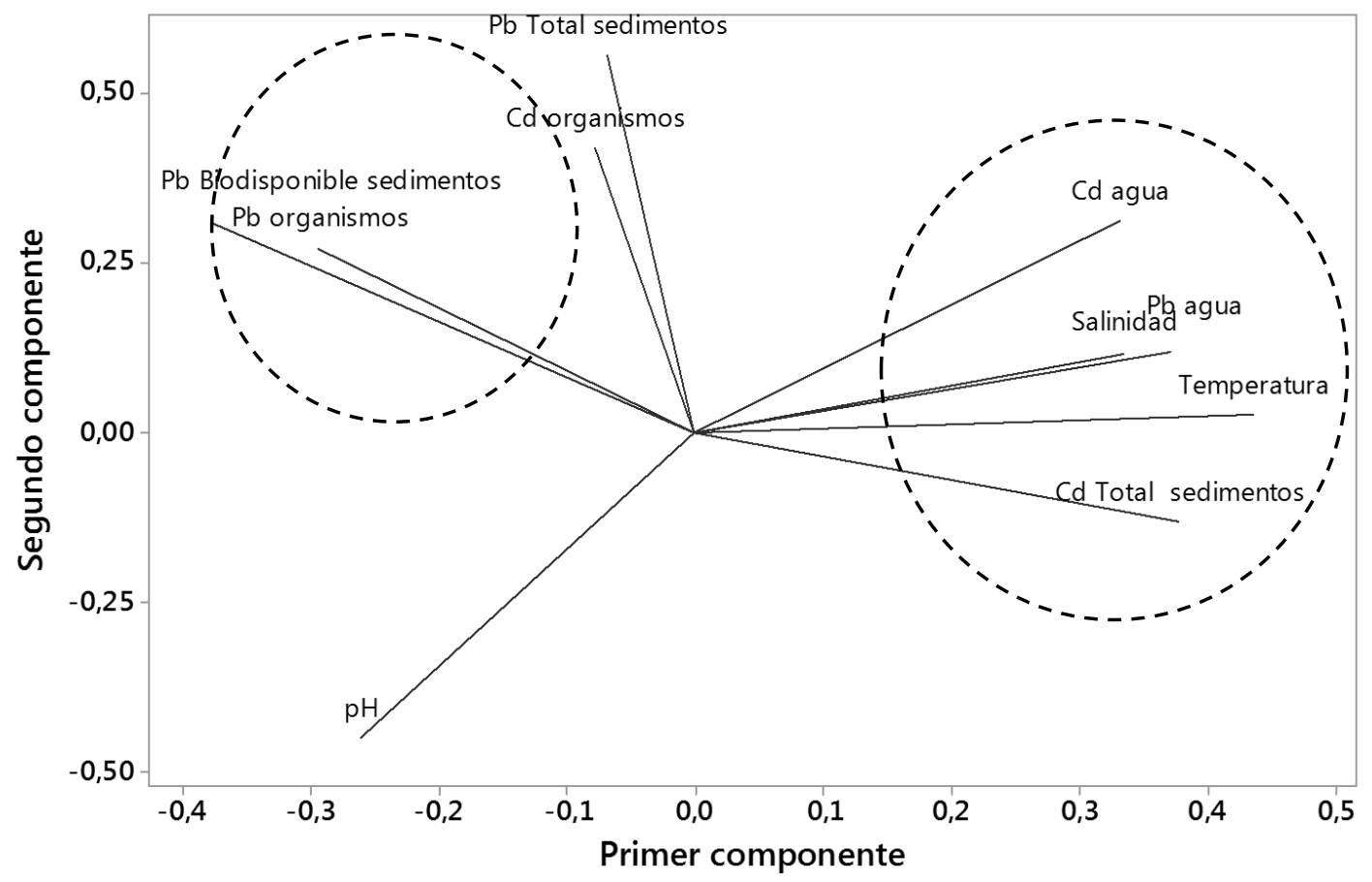

Figura 5. Análisis de componentes principales entre los parámetros fisicoquímicos y las concentraciones de los metales pesados en agua, sedimento y organismos del Estero Salado.

\section{Discusión}

Se encontraron altas concentraciones de $\mathrm{Cd}$ y $\mathrm{Pb}$ en el agua, sedimentos y organismos de la zona de estudio. Es probable que esta contaminación se xợeba a la gran cantidad de empresas que se encuentran en los alrededores del estero y que liberan sus aguas servidas al mismo sin tratamiento previo, así como al mal manejo de los desechos sólidos (EMAG, 1978; Fernández-Cárdena et al., 2014; Cárdenas y Mair, 2014; Peñafiel et al., 2017). Dentro de esas empresas se encuentran fábricas de plástico, pintura, baterías, textiles y metalmecánicas (MAE, 2017), reportadas como potenciales fuentes antropogénicas de Cd (Bjerregaard et al., 2015). Además, existen tuberías de aguas negras ilegales que llegan al estero sin ningún tipo de control (MAE, 2017).

Los dos metales se encontraron en valores superiores a los límites máximos permitidos por la normativa ambiental vigente en el Ecuador que establece los criterios de calidad admisible para la preservación de la flora y fauna en aguas dulces, fría o cálidas, y en aguas marinas y de estuarios (Acuerdo Ministerial 097A, 2015) señalando que los valores no deben sobrepasar los $0,005 \mathrm{mg} \mathrm{L}^{-1}$ de $\mathrm{Cd}$ y $0,01 \mathrm{mg} \mathrm{L}^{-1}$ de Pb. Los valores en la zona M están diez veces por encima del límite máximo para Cd y 20 veces por encima 
del límite para $\mathrm{Pb}$. En la zona $\mathrm{P}$ el $\mathrm{Cd}$ se encuentra 14 veces por encima del límite y el $\mathrm{Pb}$ 22 veces por encima del límite permitido.

Además, se determinó que las concentraciones a nivel del puente son mayores que en las zonas de manglar. Una posible explicación de la mayor concentración de metales en esta zona es que el puente se encuentra en un lugar muy transitado de la ciudad por lo que podría haber deposiciones ambientales provenientes de las emisiones de los vehículos que utilizan como combustible gasolina y diesel que pueden contener trazas de plomo. También se ha descrito que las carreteras acumulan contaminantes tales como polvo de frenos, contaminantes orgánicos y metales pesados que se transportan por escorrentía al hábitat circundante (Jones et al., 2000).

En otros estudios realizados en la misma localidad y en el mismo período de sequía, Alcívar y Mosquera (2011), no detectaron $\mathrm{Cd}$ ni $\mathrm{Pb}$ en agua en el mes de agosto, igual que Jiménez (2012), quien tampoco encontró $\mathrm{Pb}$ en agua, pero halló $0,03 \mathrm{mg} \mathrm{L}^{-1}$ de $\mathrm{Cd}$. Posteriormente, Rodríguez (2013) registraron 0.03-0,05 $\mathrm{mg} \mathrm{L}^{-1} \mathrm{de} C \mathrm{Cd}$ en agua en el Portete y en el presente trabajo se encontró entre $0,051-0,079 \mathrm{mg} \mathrm{L}^{-1}$ de $\mathrm{Cd}$ y $0,180-0,228 \mathrm{mg} \mathrm{L}^{-1}$ de $\mathrm{Pb}$ en el agua, lo que indica un incremento sustancial en la contaminación por estos metales pesados en el agua de la zona de estudio.

Dentro de las posibles consecuencias que podría traer la contaminación por cadmio y plomo del Estero Salado tenemos la afectación a las especies bentónicas, los peces, las plantas y los humanos. En algunos trabajos se ha demostrado que las especies bentónicas se ven afectadas por exponerse a elevadas concentraciones de $\mathrm{Cd}$ y $\mathrm{Pb}$ (Fanny et al., 2013). Otros estudios han demostrado que el Cd es tóxico para los organismos expuestos a este en los sedimentos (Occhiogrosso, et al., 1979; Traunspurger y Drews, 1996; Ordonez et al., 2011). Alguno de los efectos demostrados son la alteración del ciclo gametogénico de la almeja Ruditapes decussates (Smaoui-damak et al., 2006) y la reducción de la densidad de macroinvertebrados bentónicos (Ordonez et al., 2011). En los peces se han reportado numerosos efectos tóxicos del $\mathrm{Cd}$ tales como reducción significativa en su actividad natatoria (Eissa et al., 2003), aberraciones cromosómicas, núcleos anormales de células rojas y morfología anormal de la esperma (Chandra y Khunda-Bukhsh, 2004). También se han descrito efectos de los metales pesados sobre las plantas como inhibición de la germinación y el crecimiento, clorosis, estrés oxidativo (Pernía et al., 2008; Castrillo et al., 2012).

En los humanos un exceso de Cd y Pb podría generar diabetes, anemia, hipertensión, problemas cognitivos y diversos tipos de cáncer (Henson y Chedrese, 2004; Lassiter et al., 2015 ). En otra zona del Estero Salado, se ha descrito que los altos niveles de metales pesados amenazan el bienestar neurológico de la población circundante, según el Ministerio de Salud Pública quienes señalan que ha habido afectación en el aprendizaje de los niños, así como un incremento de la incidencia de cáncer de próstata, hipertensión y afecciones cardíacas (El Telégrafo, 2014).

En cuanto a los sedimentos, el $\mathrm{Cd}$ y el $\mathrm{Pb}$ superaron los LMP de la norma canadiense de $0,67 \mathrm{mg} \mathrm{kg}^{-1} \mathrm{Cd}$ y $30,2 \mathrm{mg} \mathrm{kg}^{-1} \mathrm{~Pb}$ solo en la zona del Puente. Las concentraciones encontradas en este trabajo superan significativamente las halladas por Calderón y Valdés (2012) en la bahía de San Jorge 14,96 $\pm 10,89 \mathrm{mg} \mathrm{kg}^{-1}$.

Comparativamente los valores de $\mathrm{Cd}$ encontrados en los sedimentos del Portete son menores que los reportados en otros países en zona de manglar, como Panamá 7,5 mg $\mathrm{kg}^{-1}$ (Guzmán y Jiménez, 1992), Costa Rica $7,3 \mathrm{mg} \mathrm{kg}^{-1}$ (Guzmán y Jiménez, 1992), Brasil $3,64 \mathrm{mg} \mathrm{kg}^{-1}$ (Kehrig et al., 2003), Hong Kong $3 \mathrm{mg} \mathrm{kg}^{-1}$ (Tam y Wong, 1996) y Colombia $1,92 \mathrm{mg} \mathrm{kg}^{-1}$ de Cd (Perdomo et al., 1999). Además, el Pb en sedimentos registró los valores más altos reportados hasta ahora, solo lo supera Hong Kong con $135 \mathrm{mg} \mathrm{kg}^{-1}$ (Ong Che, 1999) y Brasil con $131 \mathrm{mg} \mathrm{kg}^{-1}$ (Kehrig et al., 2003).

En cuanto a la biodisponibilidad de $\mathrm{Cd}$ en el sedimento, se observaron diferencias significativas ante la presencia de los manglares. Este hecho se ha descrito en numerosos trabajos donde señalan que los sedimentos de manglar tienen la capacidad de acumular metales pesados en formas poco disponibles y difíciles de remover debido a sus 
propiedades físicas y químicas (Tam y Wong, 1996; Espinosa et al., 2011). Estos resultados apoyan la iniciativa del Ministerio del Ambiente de reforestar el Estero con mangles, ya que como se demostró la rizosfera de los mangles ayudan a retener los metales pesados de forma no biodisponible, disminuyendo el efecto tóxico que podría generarse sobre la biota.

Sin embargo, aunque los metales se encontraban poco disponibles en la zona de manglar, algunos autores han señalado que estos metales podrían movilizarse o resuspenderse hacia la columna de agua convirtiéndose en fuentes secundarias de contaminación, si se generan cambios en las propiedades del sedimento tales como $\mathrm{pH}$, salinidad, potencial redox, contenidos de arcillas y limos y por alteración en las condiciones anaeróbicas y aeróbicas, entre otras (Tam y Wong, 1996; Espinosa et al., 2011). De allí la importancia de realizar monitoreos constantes en el Estero a fin de detectar cualquier variación en los parámetros fisicoquímicos que pudieran alterar la biodisponibilidad de los metales pesados.

Para evidenciar la biodisponibilidad de los metales en los organismos se realizó un análisis integral de la contaminación por metales pesados, donde se identificaron organismos centinelas en la zona de estudio. En este sentido, en el Estero Salado se han reportado diversas especies que presentan el potencial para ser estudiadas como bioindicadores. Dentro del Phylum Mollusca, en esta área se han reportado en orden de abundancia las especies Cerithidea varia, Cerithidea obtusa, Mytella strigata, Donax sp. y Crassostrea columbiensis (Monserrate et al., 2011). Asimismo, dentro de las especies vegetales más comunes se reportan: Rhizophora racemosa, Avicennia germinans, Conocarpus erectus y Laguncularia racemosa (Cornejo, 2014).

Sin embargo, dentro de la zona de estudio $\mathrm{P}$ no se observaron organismos, lo cual podría deberse a las altas concentraciones de $\mathrm{Cd}$ y $\mathrm{Pb}$ halladas en los sedimentos, al tipo de sustrato o la ausencia de vegetación y otros factores antrópicos. También se ha descrito que las vías terrestres generan fragmentación de ecosistemas, dispersión de especies y disminución de las poblaciones de fauna y flora nativa, así como la destrucción del hábitat natural y la reducción de la biodiversidad (Arroyave et al., 2006). En cuanto a la ausencia de los gasterópodos, Frest (2002) señala que el microclima generado por las vías terrestres es poco adecuado para estos organismos por la reducción de las sombras, aumento de la radiación solar y la temperatura.

Por el contrario, en la zona $\mathrm{M}$, se detectó la presencia de los mangles $R$. racemosa, $A$. germinans, $C$. erectus y $L$. racemosa y de los gasterópodos $L$. varia y $C$. mazatlanica. $A$ diferencia de otros trabajos realizados en el Puente Portete no se encontraron Crassostrea columbiensis (Jiménez, 2012) ni Mytella guyanensis (Rodríguez, 2013), lo que demuestra una pérdida en la diversidad que podría asociarse al incremento en la concentración de metales en agua y sedimentos. Estos resultados coinciden con los hallados por Cruz (2003) y Cárdenas-Calle y Mair (2014) quienes encontraron una reducción en la composición y abundancia de invertebrados en el Estero Salado y la atribuyen a la contaminación.

En cuanto a la capacidad de acumular metales pesados, los mangles no acumularon $\mathrm{Cd}$ ni $\mathrm{Pb}$ en sus hojas. Según algunos autores, Avicennia germinans (Campos y Gallo, 1997; González-Mendoza et al., 2007), Laguncularia racemosa (Defew et al., 2005) y Rhizophora mangle han demostrado ser acumuladoras de metales pesados (Aksoy y Öztürk, 1997; Campos y Gallo, 1997; Pinheiro et al., 2012) e incluso, Pinheiro et al., (2012), proponen el uso de las hojas de Rhizophora mangle para el monitoreo de la contaminación por metales pesados. Las especies estudiadas no acumularon $\mathrm{Pb}$ ni $\mathrm{Cd}$, probablemente debido a la nula $(\mathrm{Cd})$ o baja $(\mathrm{Pb})$ biodisponibilidad de estos metales en el sedimento, por lo que la concentración de metales en las hojas de mangle permite inferir la biodisponibilidad de estos compuestos en los sedimentos y pueden ser utilizadas como bioindicadores de biodisponibilidad.

Por el contrario, los gasterópodos $L$. varia y $C$. mazatlanica demostraron ser los mejores indicadores de contaminación, ya que acumularon $\mathrm{Cd}$ y $\mathrm{Pb}$ en altas concentraciones. Comparativamente, $L$. varia es la que acumula los metales en mayor 
proporción, sin embargo $C$. mazatlanica es más abundante por lo que se recomienda utilizarla para fines de biomonitoreo. Además, en otro estudio realizado en el Estero Salado, encontraron altas concentraciones de metales en $C$. varia $1,37-3,50 \mathrm{mg} \mathrm{kg}^{-1} \mathrm{Cd}$ y $18,73-$ $41,08 \mathrm{mg} \mathrm{kg}^{-1}$ de Pb (Alcívar y Mosquera, 2011), lo que demuestra que esta especie puede ser utilizada en el biomonitoreo del Estero Salado.

Otros trabajos demuestran que las especies de Littorina son capaces de acumular metales pesados tales como Cd, Cu, Cr, Ni y Pb (Bryan, 1983; De Wolf et al., 2000). En Korea han utilizado a la especie Littorina brevicula como bioindicadora de metales pesados obteniendo concentraciones de 0,48-27,11 $\mathrm{mg} \mathrm{kg}^{-1}$ de $\mathrm{Cd}$ y 1,41-24,9 $\mathrm{mg} \mathrm{kg}^{-1}$ de $\mathrm{Pb}$. De igual forma, en el Reino Unido, consideran a la especie Littorina littorea como biocentinela en la detección de metales en estuarios registrando concentraciones de $1,9-26 \mathrm{mg} \mathrm{kg}^{-1} \mathrm{de}$ Cd y 5,3-15,4 $\mathrm{mg} \mathrm{kg}^{-1}$ de $\mathrm{Pb}$ (Bryan, 1983).

En cuanto a los parámetros fisicoquímicos, la temperatura promedio fue mayor en la zona del Puente en comparación con la zona de Manglar. Según Arroyave et al., (2006) el efecto de borde que genera el puente crea condiciones de mayor temperatura y mayor radiación solar, lo que podría explicar la mayor temperatura del agua en la zona $\mathrm{P}$. Por el contrario, en la zona M los manglares generan sombra y reducen la temperatura. Según Rosas-Lusett et al. (2016) los manglares disminuyen en $2,5^{\circ} \mathrm{C}$ la temperatura a través de la evapotranspiración y la sombra que generan.

Además, este incremento en la temperatura en la zona $P$ podría explicar la mayor biodisponibilidad de los metales que se halló en este lugar. En este sentido, Hooda y Halloway (1993) demostraron que la biodisponibilidad del $\mathrm{Cd}$ y el $\mathrm{Pb}$ en los suelos es proporcional a la temperatura, ya que al incrementar la temperatura aumenta la solubilidad de los compuestos.

En cuanto al pH del Estero, este se encontró dentro de los límites admisibles según el AM 097A para preservación de la flora y fauna. Al comparar el pH del agua del presente estudio $(7,40-7,75)$ con trabajos anteriores realizados en el mismo lugar, los valores fueron similares: 6.5-7.8 (Alcívar y Mosquera, 2011), 6.9-7.2 (Jiménez, 2012) y 7.1-7.2 (Rodríguez, 2013). Se ha descrito que el $\mathrm{pH}$ afecta el equilibrio entre la especiación metálica, solubilidad, adsorción e intercambio de iones en el suelo y se ha considerado el factor más importante en la biodisponibilidad de los metales (McBride et al., 1997). A pH básicos los metales pesados son removidos de la solución del suelo y adsorbidos por los coloides, disminuyendo su biodisponibilidad (Alloway, 1995). En este sentido, el Estero presenta un $\mathrm{pH}$ de neutro a básico lo que reduce su disponibilidad para la biota y su potencial toxicidad.

Además, la salinidad sí registró valores superiores a los hallados en otros estudios realizados en Puente Portete, donde se determinó 21-22 \%o (Alcívar y Mosquera, 2011), 7-14\%o (Jiménez, 2012) y 10-14\%o (Rodríguez, 2013). Este incremento en la salinidad probablemente se deba a que en el presente trabajo se midió este parámetro en época de sequía y en marea baja a diferencia de los otros autores y podría justificar la mayor biodisponibilidad. Se ha demostrado que al aumentar la salinidad aumenta la biodisponibilidad del $\mathrm{Cd}$. Los cationes asociados a las sales $(\mathrm{N}, \mathrm{K})$ pueden reemplazar a los metales en los lugares de adsorción y los cloruros pueden formar complejos solubles estables tales como $\mathrm{CdCl}_{2}$ (García y Dorronsoro, 2005).

Se ha descrito que el intercambio de nutrientes y contaminantes entre los sedimentos y el agua de los sistemas estuarinos depende de diferentes parámetros físico-químicos y biológicos (De Laune et al., 1981) por lo que la biodisponibilidad de los metales pesados depende de su comportamiento químico y de los parámetros como $\mathrm{pH}$, capacidad de intercambio catiónico, presencia de cationes y aniones, óxidos de hierro, manganeso y aluminio, salinidad, contenido de arcilla y materia orgánica (Ernst, 1996; Parra y Espinosa, 2008). En este sentido, en el presente estudio no se hallaron diferencias en el pH y salinidad, por lo que se podría sugerir que las diferencias en la biodisponibilidad de metales pesados entre las zonas $\mathrm{M}$ y $\mathrm{P}$, se debe a condiciones intrínsecas de la rizosfera de los mangles. La baja disponibilidad del Cd, podría deberse a la adsorción de este metal a las arcillas del suelo y a la materia orgánica de la rizosfera de los mangles (Parra y Espinosa, 
2008). Según Guarco (2015) en el sedimento del Estero Salado en el ramal B donde se encuentra el Puente Portete existe un alto contenido de materia orgánica (8,3\%) y de arcilla $(17,19 \%)$, lo que soporta esta teoría.

Finalmente se evidenció el efecto de los parámetros fisicoquímicos sobre la biodisponibilidad de los metales y su influencia en la absorción por parte de los organismos. Se evidenció correlación entre la temperatura y la salinidad con la concentración de Cd y $\mathrm{Pb}$ en agua, donde a mayor temperatura y salinidad mayor concentración de metales en el agua. Además, se observó correlación entre el $\mathrm{Pb}$ biodisponible en el sedimento y el presente en los gasterópodos, demostrando su eficiencia como bioindicadores. De allí la importancia de realizar monitoreos integrales donde se incluyan los parámetros fisicoquímicos, la biodisponibilidad de los metales y su presencia en organismos.

\section{Conclusiones y recomendaciones}

La concentración de metales pesados en agua del Estero Salado superó los límites máximos permisibles según la normativa ambiental ecuatoriana. En la zona de manglar, los niveles de $\mathrm{Cd}$ y $\mathrm{Pb}$ se encontraron 10 y 20 veces por encima del límite máximo permisible. En la zona del puente la contaminación fue mayor registrándose valores 14 y 22 veces por encima del límite permitido según la norma ecuatoriana para $\mathrm{Cd}$ y $\mathrm{Pb}$, respectivamente.

Con respecto a los sedimentos, la zona de manglar el Cd no superó los límites establecidos, pero en la zona del puente superó 2,8 veces al límite máximo permitido para sedimentos según la Norma Internacional de Canadá. De igual manera el Pb en la zona del puente superó 5,4 veces el límite máximo permitido. Dadas las altas concentraciones de metales pesados resulta imprescindible que las autoridades tomen medidas para mitigar la contaminación y realicen monitoreos anuales a fin de evaluar periódicamente los metales en el estero y esta información pueda ser utilizada para la toma de decisiones, orientadas a la conservación y preservación de la flora y fauna.

También, en cuanto a la disponibilidad de los metales en el sedimento se evidenció que la biodisponibilidad de $\mathrm{Cd}$ se ve afectada por la presencia de los manglares ya que en la zona $\mathrm{M}$ este metal no se encontraba biodisponible, mientras que en la zona $\mathrm{P}$ por el contrario, el $\mathrm{Cd}$ se encontró biodisponible en un 70,82-89,47\%. En el caso del Pb, este se encontró biodisponible en las dos zonas. Este hallazgo apoya la teoría que los manglares son trampas para secuestrar $\mathrm{Cd}$, por lo que se debe promover la reforestación con mangles en sitios que presenten contaminación por estos metales pesados, como en el Estero Salado.

Se identificaron organismos bioindicadores de contaminación por metales pesados, llegando a la conclusión que los gasterópodos $L$. varia y $C$. mazatlanica pueden ser utilizados como bioindicadores de biodisponibilidad de $\mathrm{Cd}$ en el agua y los mangles $R$. racemosa, $A$. germinans, $C$. erectus y $L$. racemosa como especies indicadoras para detectar la biodisponibilidad en sedimentos, por lo que se recomienda realizar monitoreos integrales donde se evalúen los metales pesados en agua y sedimento y además en estos organismos bioindicadores, para tener un panorama integral de los metales en el ambiente.

\section{Agradecimientos}

Los autores agradecen al Programa Prometeo de SENESCYT por el financiamiento de los reactivos y al Ministerio del Ambiente de Ecuador (MAE) por la autorización de investigación Científica Nº15-IC-FLO/FAU-DPG/MAE. También se agradece a Thomas Andres (NY) por la revisión del texto del resumen en inglés. 


\section{Bibliografía}

Acuerdo Ministerial 097-A (2016). Acuerdo Ministerial 097-A. Quito: Ministerio de Medio Ambiente.

Aksoy, A. y Öztürk, M.A. (1997). Nerium oleander L. as a Biomonitor of Lead and Other Heavy Metal Pollution in Mediterranean Environments. Science of the Total Environment, 205 (2-3), 145-150.

Alcívar, M. y Mosquera, A. (2011). Concentración de Metales Pesados (Cr Total, Pb, Cd) En Agua Superficial y Sedimentos en el Estero Salado (Guayaquil). Tesis. Facultad de Ciencias Naturales, Universidad de Guayaquil.

Alloway, B.J. (1995) Soil processes and behaviour of metals. En: Alloway, B.J. (Ed). Heavy metals in soils. Second edition. John Wiley, New York, pp.11-37.

Arroyave, M., Gómez, C., Gutiérrez, M.E., Múnera, D., Zapata, P., Vergara, I., Andrade, L. y Ramos, K. (2006). Impacto de las carreteras sobre la fauna silvestre y sus principales medidas de manejo. Revista EIA, 5, 45-47.

Batuman, V., Maesaka, J.K., Haddad, B., Tepper, E., Landy, E. y Wedeen, R.P. (1981). The Role of Lead in Gout Nephropathy. The New England Journal of Medicine, 304 (9), 520-23.

Bjerregaard, P., Andersen, C. B. I. y Andersen, O. L. E. (2015). Ecotoxicology of MetalsSources, Transport, and Effects on the Ecosystem. Handbook on the Toxicology of Metals (Fourth Edi). Elsevier. https://doi.org/10.1016/B978-0-444-59453-2.00021-4

Bryan, G. (1983). Brown Seaweed, Fucus vesiculosus, and the Gasteropod Littorina Littoralis, as Indicators of Trace-Metal Availability in Estuaries. The Science of the Total Environment, 28, 91-104.

Burger, J. (2008). Assessment and Management of Risk to Wildlife from Cadmium. Science of the Total Environment, 389, 37-45.

Campos, N. y Gallo, M. (1997). Contenidos de Cd, Cu y Zinc en Rhizophora mangle y Avicennia germinans de La Ciénaga Grande de Santa Marta Y Bahía de Chengue, Costa Caribe Colombiana. Rev. Acad. Colomb. Cienc, XXI (79), 73-90.

Calderón, C. y Valdés, J. (2012). Contenido de metales en sedimentos y organismos bentónicos de la bahía San Jorge, Antofagasta, Chile. Revista de Biología Marina y Oceanografía, 47(1), 121-133.

Cárdenas-Calle, M. y Mair, J. (2014). Caracterización de macroinvertebrados bentónicos de dos ramales estuarinos afectados por la actividad industrial, Estero SaladoEcuador. Rev. Intropica, 9, 118-128.

Castrillo, M., Pernia, B., De Sousa, A. y Reyes, R. (2012). Utilization of Different Aspects Associated with Cadmium Tolerance in Plants to Compare Sensitive and Bioindicator Species. In Phytotechnologies: Remediation of Environmental Contaminants, Taylor and Francis, 427-440.

Chandra, P. y Khuda-Bukhsh, A.R. (2004). Genotoxic effects of cadmium chloride and azadirachtin treated singly and in combination in fish. Ecotoxicology and Environmental Safety, 58(2), 194-201.

Cornejo, X. (2014). Plants of the South American Pacific Mangrove Swamps (Colombia, Ecuador, Peru). Primera Ed., Facultad de Ciencias Naturales, Universidad de Guayaquil.

Counter, S., Buchanan, L.H. y Ortega, F. (2012). Association of Hemoglobin Levels and Brainstem Auditory Evoked Responses in Lead-Exposed Children. Clinical Biochemistry, 45 (15), 1197-1201.

Cruz, M. (2003). Malacofauna bentónica existente en los alrededores de la Ciudad de Guayaquil (Estero Salado y Río Guayas), Durante 2003. Acta Oceanográfica del Pacífico, 12(1): 135 - 145.

De Laune, R.D., Reddy, C.N. y Patrick, W.H. (1981). Effect of pH and Redox Potential on Concentration of Dissolved Nutrients in an Estuarine Sediment1. Journal of Environment Quality, 10 (3), 276. 
De Wolf, H., Backeljau, T. y Blust, R. (2000). Heavy Metal Accumulation in the Periwinkle Littorina littorea, along a Pollution Gradient in the Scheldt Estuary. Science of the Total Environment, 262, 111-21.

Defew, L.H., James, M. y Guzman, H. (2005). An Assessment of Metal Contamination in Mangrove Sediments and Leaves from Punta Mala Bay, Pacific Panama. Marine Pollution Bulletin, 50, 547-52.

Eissa, B.L., Salibiani, A., Ferrari, L., Porta, P. y Borgnia, M. (2003). Evaluación toxicológica no invasiva del cadmio: modificaciones de biomarcadores conductuales en Cyprinus carpio. Biología Acuática, 20, 56-62.

El Telégrafo. (2014). Metales pesados dejan efectos neurológicos en vecinos del Estero. Recuperado de https://www.eltelegrafo.com.ec/noticias/guayaquil/10/metales-pesadosdejan-efectos-neurologicos-en-vecinos-del-estero

EMAG. (1978). Recuperación del Estero Salado. Plan de Trabajo. Empresa Municipal de Alcantarillado de Guayaquil. Guayaquil, Ecuador . 43 pp.

Ernst, W.H.O. (1996). Bioavailability of Heavy Metals and Decontamination of Soils by Plants. Applied Geochemistry, 11 (1-2), 163-67.

Espinosa, L., Parra, J. y Villamil, C. (2011). Determinación del Contenido de Metales Pesados en Las Fracciones Geoquímicas del Sedimento Superficial Asociado a Los Manglares de la Ciénaga Grande de Santa Marta, Colombia. Bol. Invest. Mar. Cost., $40(1), 7-23$.

Fanny, C., Virginie, A., Jean-François, F., Jonathan, B., Marie-Claude, R. y Simon, D. (2013). Benthic indicators of sediment quality associated with run-of-river reservoirs. Hydrobiologia, 703(1), 149-164.

Fernández-Cadena J.C., Andrade, S., Silva-Coello, C.L. y De la Iglesia, R. (2014). Heavy Metal Concentration in Mangrove Surface Sediments from the North-West Coast of South America. Marine Pollution Bulletin, 82 (1-2), 221-226.

Frest, T.J. (2002). Native snails: Indicators of Ecosystem Health. p. 211-215, in: Welfare Ranching, G. Wuerthner and M. Matteson (Eds.). Island Press, Sausalito, California.

Gallagher, C.M. y Meliker, J.R. (2010). Blood and Urine Cadmium, Blood Pressure, and Hypertension: A Systematic Review and Meta-Analysis. Environmental Health Perspectives, 118 (12), 1676-84.

García, I. y Dorronsoro, C. (2005). Contaminación por metales pesados. En: Tecnología de Suelos. Universidad de Granada. Departamento de Edafología y Química Agrícola. Obtenido de http://edafologia.ugr.es/conta/tema15/introd.htm.

González-Mendoza, D., Ceja-Moreno, V., Gold-Bouchot, G., Escobedo-Graciamedrano, R.M., Del-Rio, M., Valdés-Lozano, D. y Zapata-Pérez, O. (2007). The Influence of Radical Architecture on Cadmium Bioaccumulation in the Black Mangrove, Avicennia Germinans L. Chemosphere, 67 (2), 330-34.

Guarco, K. (2015). Tratamiento del sedimento marino del ramal B del Estero Salado para la Remoción de metales pesados. Tesis de Grado de la la Facultad de Ciencias Naturales y Matemática. Escuela Superior Politécnica del Litoral Obtenido de: http://www.dspace.espol.edu.ec/xmlui/handle/123456789/31957

Guzmán, H.M. y Jiménez, C. (1992). Contamination of Coral Reefs by Heavy Metals along the Caribbean Coast of Central America (Costa Rica and Panama). Marine Pollution Bulletin, 24 (11), 554-61.

Henson, M.C. y Chedrese, P. (2004). Endocrine Disruption by Cadmium, a Common Environmental Toxicant with Paradoxical Effects on Reproduction. Experimental Biology and Medicine, 229 (5), 383-92.

Hooda, P.S. y Alloway, B.J. (1993). Effect of time and temperatura on the bioavailability of $\mathrm{Cd}$ and $\mathrm{Pb}$ from sludge-amended soil. J. Soil. Sci., 44, 97-110.

Järup, L. y Alfvén, T. (2004). Low Level Cadmium Exposure, Renal and Bone Effects--the OSCAR Study. Biometals, 17 (5), 505-509.

Jiménez, D. (2012). Cuantificación de Metales Pesados (cadmio, cromo, níquel y plomo) en agua superficial, sedimento y organismos (Crassostrea Columbiensis) Ostión de 
mangle en el Puente Portete Del Estero Salado (Guayaquil). Tesis. Facultad de Ciencias Naturales, Universidad de Guayaquil.

Jones, J.A., Swanson, F.J., Wemple, B.C. y Snyder, K.U. (2000). Effects of roads on hydrology, geomorphology, and disturbance patches in stream networks. Conservation Biology 14(1), 76-85.

Julin, B., Wolk, A., Johansson, J.E., Andersson, S.O., Andrén, O. y Akesson, A. (2012). Dietary Cadmium Exposure and Prostate Cancer Incidence: A Population-Based Prospective Cohort Study. British Journal of Cancer, 107, 895-900.

Kehrig, H.A., Pinto, F.N., Moreira, I., y Malm, O. (2003). Heavy Metals and Methylmercury in a Tropical Coastal Estuary and a Mangrove in Brazil. Organic Geochemistry, 34 (5), 661-69.

Lacerda, L.D., Martinelli, L.A., Rezende, C.E., Mozeto, A., Ovalle, A.R., Victoria, R.L., Silva, C.A. y Nogueira, F.B. (1988). The Fate of Trace Metals in Suspended Matter in a Mangrove Creek during a Tidal Cycle. The Science of the Total Environment, 75, 16980.

Lassiter, M., Owens, E., Patel, M.M., Kirrane, E., Madden, M., Richmond-Bryant, J., Hines, E., Davis, A., Vinikoor-Imler, L. y Dubois, J.J. (2015). Cross-species coherence in effects and modes of action in support of causality determinations in the U.S. Environmental Protection Agency's Integrated Science Assessment for Lead. Toxicology, 330, 19-40.

MAE. (2017). Listado de industrias con descargas al Estero Salado. Recuperado de http://www.ambiente.gob.ec/wp-content/uploads/downloads/2012/08/matriz industriasEMPRESAS.pdf

McBride, M., Sauve, S. y Hendershot, W. (1997). Solubility control of Cu, Zn, Cd and Pb in contaminated soils. Eur. J Soil Sci, 48, 337-346.

McElroy, J., Shafer, M.M., Trentham-Dietz, A., Hampton, J.M. y Newcomb, P. (2006). Cadmium Exposure and Breast Cancer Risk. Journal of the National Cancer Institute, 98 (12), 869-73.

Mero, M., Arcos, V., Egas, F., Siavichay, F. y Lindao, G. (2012). Determinación de Metales Pesados ( $\mathrm{Cd}$ y $\mathrm{Pb}$ ) en moluscos bivalvos de interés comercial Anadara tuberculosa y A. grandis de Puerto El Morro, Ecuador. Revista de Divulgación de la Dirección de Investigaciones y Proyectos Académicos de la Universidad de Guayaquil, 4 (4), 1932.

Monserrate, L., Medina, J. y Calle, P. (2011). Estudio de condiciones físicas, químicas y biológicas en la zona intermareal de dos sectores del Estero Salado con diferente desarrollo urbano. Tesis. Escuela Superior Politécnica del Litoral Guayaquil. Recuperado de: http://www.dspace.espol.edu.ec/handle/123456789/19190.

Mushak, P. (2011). Lead and Public Health: Science, Risk and Regulation. Retrieved from https://books.google.com/books?id=T5HcahQSOfACypgis $=1$.

Occhiogrosso, T. J., Waller, W. T. y Lauer, G. J. (1979). Effects of heavy metals on benthic macroinvertebrate densities in foundry cove on the Hudson river. Bulletin of Environmental Contamination and Toxicology, 22(1), 230-237.

Ong Che, RG. (1999). Concentration of 7 Heavy Metals in Sediments and Mangrove Root Samples from Mai Po, Hong Kong. Marine Pollution Bulletin, 39 (1-12), 269-279.

Ordonez, C., Lougheed, V. L., Gardea-torresdey, J. L. y Bain, L. J. (2011). Impact of Metals on Macroinvertebrate Assemblages in the Forgotten Stretch of the Rio Grande. Archives of Environmental Contamination and Toxicology. 1992, 426 - 436.

Parra, J. y Espinosa, L. (2008). Distribución de metales pesados (Pb, Cd y Zn) en perfiles de sedimento asociado a Rhizophora mangle en el Río Sevilla, Cienaga Grande de Santa Marta, Colombia. Bol. Invest. Mar. Cost., 37 (1), 95-110.

Peñafiel, M., Vallejo, A. y Chalen, J. (2017). Evaluación de la calidad físico-química en agua y sedimentos del estero salado en el noroeste de América Latina. Pol. Con. 2(6), 641 669. 
Perdomo, L., Ensminger, I., Espinosa, L., Elster, C., Wallner-kersanach, M. y Schnetter, ML. (1999). The Mangrove Ecosystem of the Ciénaga Grande de Santa Marta (Colombia): Observations on Regeneration and Trace Metals in Sediment. Marine Pollution Bulletin, 37 (8-12), 393-403.

Pernía, B., De Sousa, A., Reyes, R. y Castrillo, M. (2008). Biomarcadores de contaminación por cadmio en plantas. Interciencia, 33, 112-119.

Pinheiro, M., Gándara, P., Silva, E., De Almeida, L.F., Almeida, A.A. y Pinheiro, F. (2012). Accumulation of six metals in the mangrove Crab Ucides cordatus (Crustacea: Ucididae) and its food source, the red mangrove Rhizophora mangle (Angiosperma: Rhizophoraceae). Ecotoxicology and Environmental Safety, 81, 114-121.

Poma, P. (2008). Lead Effects on Humans. Anales de La Facultad de Medicina, 69 (2), 120-126.

Revis, N.W., Zinsmeister, R. y Bull, R. (1981). Atherosclerosis and Hypertension Induction by Lead and Cadmium lons: An Effect Prevented by Calcium Ion. Proceedings of the National Academy of Sciences of the United States of America, 78 (10), 6494-6498.

Richardson, M.E., Fox, D. y Fry, B.E. (1974). Pathological Changes Produced in Japanese Quail by Ingestion of Cadmium. The Journal of Nutrition, 104, 323-338.

Rodríguez, F. (2013). Cuantificación de cadmio, plomo y níquel en agua superficial, sedimento y Organismos (Mytella Guyanensis) en los puentes Portete Y 5 de Junio Del Estero Salado (Guayaquil). Tesis, Facultad de Ciencias Naturales, Universidad de Guayaquil.

Rosas-Lusett, M.A., Bartorila, M.A. y Ocón-Morales, S. (2016). Laguna del Carpintero, regulador climático en el área urbana de Tampico, Tamaulipas, México. Legado de Arquitectura y Diseño, 20, 113-124.

Smaoui-Damak, W., Rebai, T., Berthet, B. y Hamza-Chaffai, A. (2006). Does cadmium pollution affect reproduction in the clam Ruditapes decussatus? A one-year case study. Comparative Biochemistry and Physiology Part C: Toxicology \& Pharmacology, 143(2), 252-261.

Schwartz, G.G., Il'Yasova, D. y Ivanova, A. (2003). Urinary Cadmium, Impaired Fasting Glucose, and Diabetes in the NHANES III. Diabetes Care, 26 (2), 468-470.

Suwazono, Y., Sand, S., Vahter, M., Filipsson, A. F., Skerfving, S., Lidfeldt, J. y Åkesson, A. (2006). Benchmark Dose for Cadmium-Induced Renal Effects in Humans. Environmental Health Perspectives, 114(7), 1072-1076.

Tam, N.F.Y. y Wong, Y.S. (1996). Retention and distribution of heavy metals in mangrove soils receiving wastewater. Environmental Pollution, 94 (3), 283-291.

Traunspurger, W. y Drews, C. (1996). Toxicity analysis of freshwater and marine sediments with meio- and macrobenthic organisms: a review. Hydrobiologia, 328(3), 215-261.

Vásquez, R., Bastardo, A. y Mundarain, I.K. (2005). Ensayo de Toxicidad Aguda CL50-96h con acetato de cadmio y parámetros hematológicos en el híbrido cultivado Colossoma macropomum X Piaractus brachypomus. Zootecnia Tropical, 23 (3), 247257.

WHO. (2010). Exposure to Cadmium: A Major Public Health Concern. World Health Organization, 3-6. Retrieved from <http://www.who.int/ipcs/features/cadmium.pdf. > 